\title{
CHANGES IN INCIDENCE OF FEBRILE NEUTROPENIA (FN) HOSPITALIZATION FOLLOWING MYELOSUPPRESSIVE CHEMOTHERAPY IN MEDICARE, 2010-2017
}

\author{
Xian Shen', Manasi Suryavanshi', Iman Mohammadi', Rajesh Belani², Prasad Gawade ${ }^{3}$
}

1: Avalere, Inc; 2: US Medical, Amgen, Inc. Thousand Oaks, CA; 3: Center for Observational Research, Amgen, Inc. Thousand Oaks, CA.

\section{INTRODUCTION}

- In the era of targeted therapies and immunotherapies, conventional myelosuppressive chemotherapy continues to play an important role for most patients.

- Febrile neutropenia (FN) is a serious complication associated with increased morbidity and mortality among patients receiving myelosuppressive chemotherapy.

-When neutropenic patients develop fever (i.e., FN), the likelihood of infection and serious consequences often necessitates prolonged hospitalization for ongoing monitoring, and administration of intravenous antibiotics.

- Use of myelosuppressive chemotherapy is evolving but there is currently no data describing changes over time in incidence of FN hospitalization following initiation of myelosuppressive chemotherapy.

\section{METHODS}

- Study Period: 1/1/09 - 12/31/17

- Baseline Period: 1/1/09 - 12/31/09

\section{Study Population:}

- Overall cohort of new users of myelosuppressive chemotherapy defined as patients initiating myelosuppressive agent for the first time during the study period (i.e. index date) with no use of any myelosuppressive agents 12 months prior to the index date.

- Patients were required to have 12 months of continuous enrollment in Medicare Part A and Part B prior to the index date.

- Annual overall cohort of all patients, and patients diagnosed with breast, lung or colon cancer, non-Hodgkin's lymphoma (NHL), or ovarian cancer and initiating myelosuppressive chemotherapy between 1/1/10 - 11/30/17 were created.

- For selected tumor type, patients were required to have $\geq 1$ diagnosis claim in inpatient setting or $\geq 2$ diagnosis claims ( $\geq 7$ days apart) in outpatient setting within 1 year prior or 1 month after the index date.

\section{Data Source}

- 100\% Medicare Part A \& B claims

\section{METHODS (continued)}

- List of myelosuppressive agents was created by an oncologist and myelosuppressive chemotherapy use was identified using hospital outpatient or physician claims.

\section{Follow-up}

- Patients were followed from index date to the earliest of 30 days, disenrollment, or death

- FN was assessed during the first 30 days of follow-up

- 2 definitions were used to identify FN (because of the lack of specific ICD-9 code for FN)

- Sensitive definition: $\geq 1$ inpatient diagnosis claims for neutropenia or fever or infection in any position.

- Specific definition: $\geq 1$ inpatient diagnosis claims for neutropenia in any position.

\section{Statistical Analysis}

- Incidence proportion of FN hospitalization was calculated as percentage and $95 \%$ confidence interval (binomial) stratified by tumor type for annual cohorts

\section{RESULTS}

- A total of 1,151,313 patients met study inclusion criteria for overall cohort including breast ( $n=142,611)$, lung $(n=261,564)$, and colon cancer $(n=107,644), \mathrm{NHL}(\mathrm{n}=45,702)$, and ovarian cancer $(n=40,287)$.

- There was slight decrease in incidence of FN hospitalization (sensitive definition) from 2010 to 2017 for the overall cohort (9.5 to $8.6 \%$; Fig 1) and all tumors, breast (8.8 to $8.5 \%$ ), lung (13.8 to $12.3 \%$ ), colon (7.6 to $7.2 \%$ ), and NHL (19.8 to 16.8\%), except ovarian (9.4 to $9.9 \%$ ) (Fig 3 ).

- In contrast, the incidence of FN hospitalization (specific definition) was stable from 2010 to 2017 for the overall cohort (2.8 to 2.8\%; Fig 2) with slight increase observed for breast (4.5 to $5.0 \%$ ), lung (4.0 to $4.4 \%$ ), colon (2.0 to $2.8 \%$ ), and ovarian cancer (2.9 to $4.5 \%$ ) with inverted U observed for NHL (10.3 to 9.9\%), (Fig 3).

\section{RESULTS (continued}

- As expected, the FN hospitalization incidence rates are higher across all tumor types when using the sensitive definition for FN hospitalization as compared to the specific definition (Fig 3).
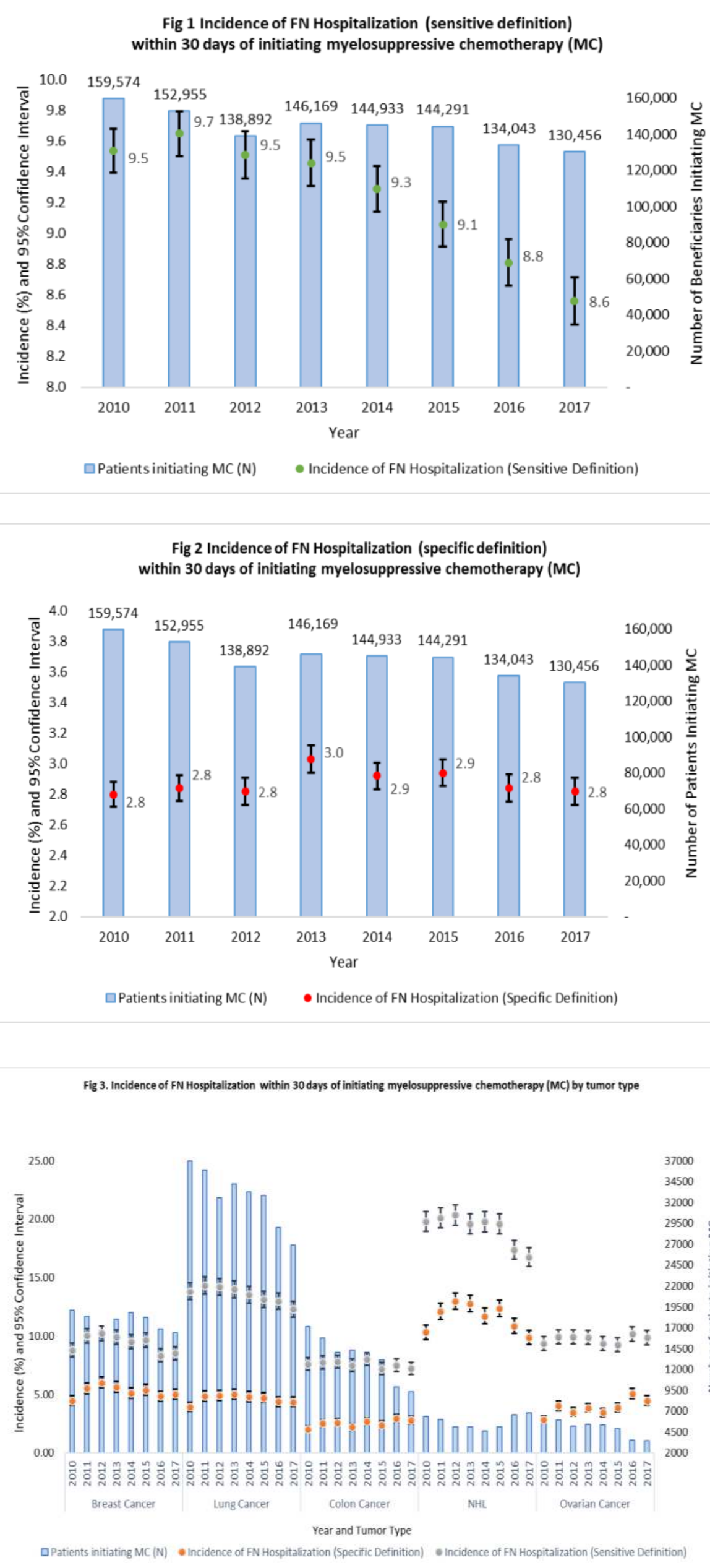

\section{CONCLUSIONS}

- There is a slight decrease in incidence of FN hospitalization identified using sensitive definition. However, the incidence of FN hospitalization appears unchanged using the specific definition.

- Despite the guidelines for using myeloid growth factors and changes in myelosuppressive chemotherapy, hospitalization for febrile neutropenia continues to be a burden for cancer patients across multiple tumor types 